\title{
HIGH HEAT FLUX PERFORMANCE OF PLASMA FACING MATERIALS AND COMPONENTS UNDER SERVICE CONDITIONS IN FUTURE FUSION REACTORS
}

\author{
Jochen Linke \\ Forschungszentrum Jülich GmbH, IEK-2, EURATOM Association, D-52425 Jülich, Germany, j.linke@fz-juelich.de
}

\begin{abstract}
The first wall and the divertor in present-day or next step thermonuclear fusion devices are exposed to intense fluxes of charged and neutral particles, in addition the plasma facing materials and components are subjected to radiation in a wide spectral range. These processes, in general referred to as 'plasma wall interaction' will have strong influence on the plasma performance, and moreover, they have major impact on the degradation and on the lifetime of the plasma facing armour and the joining interface between the plasma facing material and the heat sink. Beside physical and chemical sputtering processes, thermal fatigue damage due to cyclic heat fluxes during normal operation and intense thermal shocks caused by severe thermal transients are of serious concern for the engineers which develop reliable wall components. In addition, the material and component degradation due to high fluxes of energetic neutrons is another critical issue in D-T-burning fusion devices which requires further extensive research activities. This paper represents a tutorial focussed on the development and characterization of plasma facing components for thermonuclear fusion devices [1].
\end{abstract}

\section{INTRODUCTION}

The plasma facing components in magnetic confinement experiments, i.e. the first wall (FW), the limiters and the divertor will be exposed to intense thermal loads during plasma operation. In existing and next step devices the resulting thermal loads to the first wall will in general remain below $1 \mathrm{MWm}^{-2}$. However, special attention has to be paid to high heat flux components, i.e. to the limiter and the divertor with power densities up to approximately $10 \mathrm{MWm}^{-2}$ for next step tokamaks (such as ITER) or stellarators (e.g. Wendelstein 7-X) during normal plasma operation. These requirements make high demands on the selection of qualified materials and reliable fabrication processes for actively cooled plasma facing components [2 - 3].

Beside the above mentioned quasi-stationary heat loads, short transient thermal pulses with deposited energy densities in the order of several ten $\mathrm{MJm}^{-2}$ are a serious concern for next step tokamak devices, in particular for ITER. The most serious of these events are plasma disruptions. Here a considerable fraction of the plasma energy is deposited on a localized surface area in the divertor strike zone region; the time scale of these events is typically in the order of milliseconds. In spite of the fact that a dense cloud of ablation vapour will form above the strike zone, only partial shielding of the divertor armour from incident plasma particles will occur. As a consequence, thermal shock induced crack formation, vaporization, surface melting, melt layer ejection, and particle emission induced by brittle destruction processes will limit the lifetime of the components. In addition, dust particles (neutron activated or toxic metals or tritium enriched carbon) are a serious concern form a safety point of view. Instabilities in the plasma positioning (vertical displacement events, VDE) also may cause irreversible damage to plasma facing components, particularly to metallic wall armour.

Furthermore, irradiation induced material degradation due to the impact with $14 \mathrm{MeV}$ neutrons in D-T-burning plasma devices is another critical issue, both, from a safety point of view, but also under the aspect of the component lifetime. Next step thermonuclear confinement devices such as ITER with an integrated neutron fluence in the order of 1 dpa (displacements per atom; for low-Z materials $1 \mathrm{dpa}$ corresponds to approx. $10^{25} \mathrm{n}^{-2}$ ) do not pose any unsolvable material problems. However, in future devices such as DEMO or in commercial fusion reactors with integrated neutron wall loads of 80 to 150 dpa new radiation resistant materials have to be developed and tested under realistic conditions. Due to the lack of an intense 14 $\mathrm{MeV}$ neutron source, complex neutron irradiation experiments are been performed in material test reactors to quantify the n-induced material damage. These tests provide the required data base on the degradation of thermal and mechanical parameters; in addition the thermal fatigue and thermal shock performance of irradiated high heat flux components is another important issue for the engineering design, the licensing and the safe operation of future fusion reactors.

\section{DEVELOPMENT AND MANUFACTURING OF PLASMA FACING COMPONENTS}

The applicability of a future energy generating fusion reactors is, among others, based on the feasibilities of plasma facing components which can guarantee a reasonable lifetime from a safety and economical point of view. This lifetime is limited mainly by thermal fatigue due to cyclic thermal loads and by thermally induced mechanical stresses to these components [4]. Transient 
thermal loads, in particular tokamak specific plasma disruptions can deposit energy densities of several ten $\mathrm{MJm}^{-2}$. These events have pulse durations in the order of 1 millisecond and will damage and/or erode the divertor, especially in the separatrix strike zone region. Further transient events which deposit a large fraction of the plasma energy on relatively small wall areas are the vertical displacement events (VDE).

The quasi-continuous plasma operation in large future confinement experiments is associated with another transient heat load event, namely energy deposition by type-I ELMs (edge localized modes) which will deposit another non-negligible amount of energy during each event; the expected power deposition is in the order of GW per square metre on a sub-millisecond time scale. Up to now only limited information is available on the material performance under these events. However, there is a serious concern that high cycle fatigue damage and thermal erosion combined with brittle destruction (BD) might be another lifetime limiting event.

The expected loading scenarios for the above mentioned thermal fatigue and thermal shock loads are strongly design dependent. The expected thermal loads are shown schematically in table 1 for the stellarator W 7-X, for the ITER design, and for a future thermo-nuclear fusion reactor. It should be noted that the intense flux of $14 \mathrm{MeV}$ neutrons will additionally degrade all (plasma facing and structural) materials in D-T burning devices such as ITER or the reactor; this is subject of an extensive long-term materials test programme.

TABLE I. Wall loading in Wendelstein 7-X, in ITER and in a thermonuclear fusion reactor

\begin{tabular}{|c|c|c|c|}
\hline & W7-X & ITER & reactor \\
\hline heat flux FW $/ \mathrm{MWm}^{-2}$ & $<0.2$ & $<1$ & $<1$ \\
\hline heat flux divertor $/ \mathrm{MWm}^{-2}$ & 10 & $5-20$ & $\approx 5-20$ \\
\hline VDEs $/ \mathrm{MJm}^{-2}$ & $?$ & 60 & - \\
\hline disruptions $/ \mathrm{MJm}^{-2}$ & - & $\approx 10$ & - \\
\hline ELMs $/ \mathrm{MJm}^{-2}$ & $?$ & 1 & $?$ \\
\hline neutron fluence $/ \mathrm{dpa}$ & - & $<1$ & $<150$ \\
\hline
\end{tabular}

The technical design solutions which are considered today for the plasma facing components in the ITER divertor (cf. Fig. 1) are mainly based on carbon or refractory metals as plasma facing materials (PFM) and copper alloys for the heat sink. The selection of these materials [5] was based on a number of criteria; the most critical requirements are summarizes in Fig. 2.

The prime candidate for the first wall region is the low$\mathrm{Z}$ material beryllium. Due to its affinity to oxygen it is an excellent getter material which guarantees plasma discharges with low impurity levels; compared to carbon, it also exhibits better erosion resistance and hence, a reduced material transport during plasma operation of the fusion device. In addition, Be is characterized by a rather good thermal conductivity $\left(\approx 190 \mathrm{Wm}^{-1} \mathrm{~K}^{-1}\right.$ at $\mathrm{RT}$ ) to remove the surface heat flux and to avoid overheating of the wall structures. This is most essential, in particular for a first wall made from beryllium tiles or beryllium coatings which exhibit only a moderate melting point of about $1285^{\circ} \mathrm{C}$ (see Fig. 3).

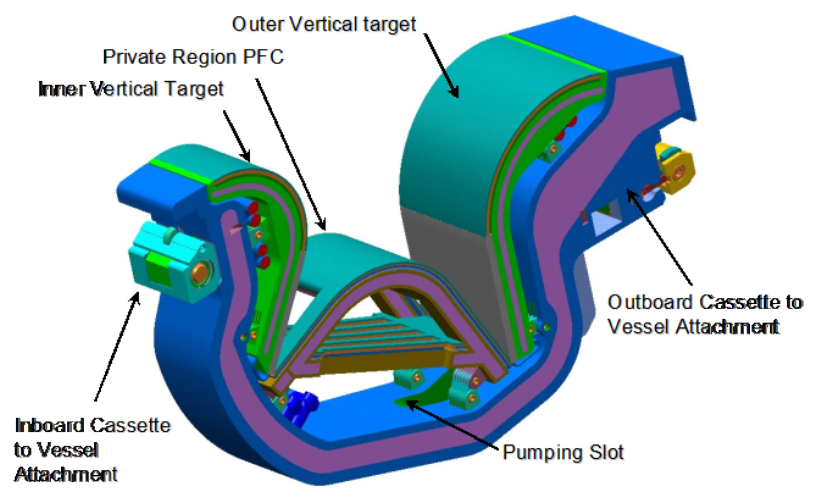

Fig. 1 Divertor cassette for ITER

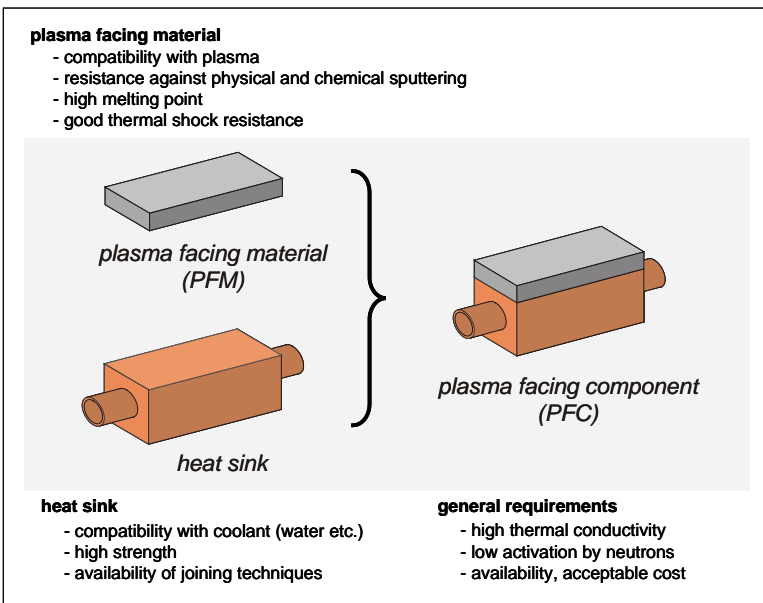

Fig. 2 Requirements for plasma facing and heat sink materials [10]

The divertor region and the baffle components in ITER will be manufactured from tungsten and/or carbon-fibrecomposites (CFC) with integrated coolant structures. Compared to beryllium, tungsten is a refractory metal with an extremely high melting point $\left(3410^{\circ} \mathrm{C}\right)$ and an adequate room temperature thermal conductivity of approx. $140 \mathrm{Wm}^{-}$ ${ }^{1} \mathrm{~K}^{-1}$; hence from a thermo-physical point of view tungsten appears to be the most attractive material candidate for high heat flux component. A drawback however is its brittle nature; tungsten is ductile and easily machinable only above the so-called ductile-brittle-transition-temperature 
(DBTT) of about $400^{\circ} \mathrm{C}$. In addition tungsten shows a strong tendency to recrystallize at high temperatures well below the melting point. Compared to the other PFM candidates (Be or $\mathrm{C}$ ) tungsten is significantly activated during neutron irradiation.

\begin{tabular}{|c|c|c|c|}
\hline & $\mathrm{Be}$ & CFC & $\mathbf{W}$ \\
\hline atomic number $Z$ & 4 & 6 & 74 \\
\hline $\begin{array}{l}\text { max. allowable concentration } \\
\text { in the plasma }\end{array}$ & $15 \%$ & $12 \%$ & $1 \mathrm{ppm}$ \\
\hline $\begin{array}{c}\text { thermal conductivity } \lambda \\
{[\mathrm{W} / \mathrm{mK}]}\end{array}$ & 190 & $200 \ldots 500$ & 140 \\
\hline melting point $\left[{ }^{\circ} \mathrm{C}\right]$ & 1285 & $\begin{array}{l}3500 \\
\text { (subl.) }\end{array}$ & 3410 \\
\hline $\begin{array}{l}\text { coefficient of thermal } \\
\text { expansion }\left[10^{-6} \mathrm{~K}^{-1}\right]^{*}\end{array}$ & 11.5 & $\sim 0$ ** & 4.5 \\
\hline n-irradiation behaviour & swelling & $\begin{array}{l}\text { decrease } \\
\quad \text { in } \lambda\end{array}$ & $\begin{array}{c}\text { activa } \\
\text { tion }\end{array}$ \\
\hline${ }^{*} \mathrm{CTE}$ copper $=16 \cdot 10^{-6} \mathrm{~K}^{-1}$ & \multicolumn{3}{|c|}{${ }^{* *}$ NB31 in pitch fiber direction } \\
\hline
\end{tabular}

Fig. 3 Thermo-physical properties of metallic and carbon based plasma facing materials.

The third candidate material, carbon, is of special importance since it does not melt. This special feature makes carbon an attractive candidate for the strike zone of the so-called separatrix on the divertor. Here it can withstand very high heat loads without the risk of forming any liquid phase; however, sublimation of carbon at elevated temperatures $\left(\mathrm{T} \geq 2200^{\circ} \mathrm{C}\right)$ is becoming essential and an enhanced material erosion due to brittle destruction (cf. chapter 5) is also of serious concern. On the other hand, the thermo physical properties are excellent, in particular if fibre-reinforced grades are taken into consideration. Depending on the selected fibre type and the weave geometry, these carbon-fibre reinforced carbons can be manufacture to day with thermal conductivities equal or even better compared to copper (up to $\approx 500 \mathrm{Wm}^{-1} \mathrm{~K}^{-1}$ ). However, this excellent thermal conductivity will be degraded rapidly under the influence of thermal or fast neutrons (cf. chapter 6). The fibre reinforcement will also improve the strength of the composite in comparison to conventional isotropic fine grain graphites which are frequently used as plasma facing armour in present-day tokamaks or stellarators.

Different design options for the attachment of the plasma facing material to the heat sink (cf. Fig. 4) have been developed, manufactured and tested $[6,7,9]$. The heat sink, in general a precipitation hardened or a dispersion strengthened copper-alloy with an integrated high pressure coolant tube has now become the standard technology for ITER or other existing medium- and long-pulse fusion devices. To reduce stresses which might affect the integrity of the plasma facing material or the joint (stresses due to the mismatch between the plasma facing and the heat sink material, as well as thermally induced stresses due to the thermal gradient during plasma exposure), a segmentation of the PFM using thin slots perpendicular to the surface down to the heat sink (so-called castellations, or 'macrobrush') is frequently used. To guarantee a non-detachable contact between the PFM and the heat sink a number of joining techniques such as brazing, hot isostatic pressing (HIPing), electron beam welding or diffusion bonding have been developed and applied successfully [8 - 10].
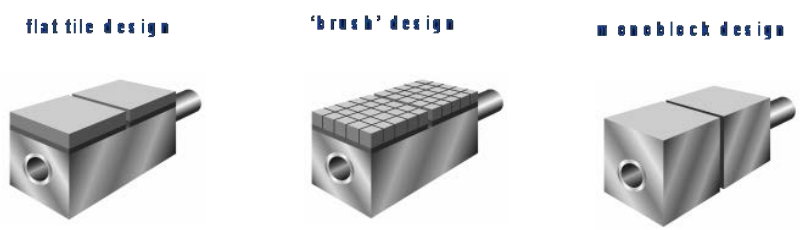

Fig. 4 Design options for actively cooled plasma facing components for divertor applications [9]

The interface between the PFM and the heat sink does not necessarily require a flat geometry; the so-called monoblock design (also: 'tube in block' solution) consists of cube-shaped monolithic tiles which are equipped with a cylindrical hole which allows the joining to the water cooled copper tube directly. This design option has an unrivalled advantage since the loss of the PFM-tile under thermal excursions which might be associated with a major failure of the whole component (cascade failure [11]) is excluded.

\section{CHARACTERIZATION OF PLASMA FACING MATERIAL AND COMPONENTS}

To select among different commercially available plasma-facing and heat sink materials a reliable characterization is necessary. An extensive data base is essential to choose the best suitable material candidates and the applied treatments (alloying, thermo-mechanical treatments such as heat exposure, rolling or forging, sintering etc.). In addition, a number of promising new materials have been developed and improved in a laboratory scale and are now ready for an upgrading for an industrial production. To identify the most promising candidates and later, to guarantee the material parameters during the serial production, an extensive set of different characterization techniques is prerequisite for the successful development and manufacturing of reliable high heat flux components. This material characterization must cover the full temperature range for the particular application; in addition, a number of material properties are also required in the neutron irradiated state to predict the material and component performance during nuclear operation of a thermonuclear facility such as ITER. This characterization is not limited to the materials; the integrity of joints has to be evaluated and demonstrated by reliable techniques (X- 
ray methods, ultrasonic inspection, infra-red techniques to identify imperfections or thermal barriers in the interface layer) [17].

Material characterization also includes the testing of actively cooled components under fusion specific loading conditions. A number of test facilities suitable to provide thermal loads with power densities ranging from the $\mathrm{MW} / \mathrm{m} 2$ to several $\mathrm{GW} / \mathrm{m} 2$, and pulse durations ranging from a few hundred microseconds to almost continuous power loading have been developed in several laboratories world wide [12]. Most of these test devices (so-called high heat flux (HHF) test facilities) are based on intense electron or hydrogen ion beams which are used in pulsed and/or scanned modes to simulate the thermal loads which are expected during normal operation scenarios; short thermal pulses are applied to characterize the material or component performance under normal (ELMs) or off-normal events (VDEs, disruptions). For the latter type of HHF-experiment also plasma accelerators [18] and ion beam facilities play an important role.

\section{THERMAL FATIGUE BEHAVIOUR OF PLASMA FACING COMPONENTS}

To evaluate the thermo mechanical performance of various divertor designs, a significant number of small scale divertor components have been manufactured by industry or research laboratories. These cover different design options (flat tile, monoblock) and different joining techniques for both, CFC and tungsten armour [4]. In the following a survey of selected plasma facing component with CFC and tungsten armour for the divertor and with beryllium coatings/tiles for first wall applications are summarized; the major characteristics of carbon and tungsten armoured modules and the results for medium term thermal fatigue tests are listed in Fig. 5.

The heat flux limits which have been obtained so far in electron beam experiments on small scale mock-ups with typical cycle number of $n=1000$ can be summarized as follows:

- CFC flat tiles withstood cyclic thermal loads up to $19 \mathrm{MWm}^{-2}$,

- CFC monoblocks have been tested up to $25 \mathrm{MWm}^{-2}$,

- tungsten flat tiles (macrobrush design) didn't show any failure up to $18 \mathrm{MWm}^{-2}$,

- tungsten monoblocks (drilled W-tiles and W-lamellae) withstood up to $20 \mathrm{MWm}^{-2}$.

These data show very clearly that technical solutions for the divertor targets are feasible which meet or even exceeded the HHF requirements for ITER.

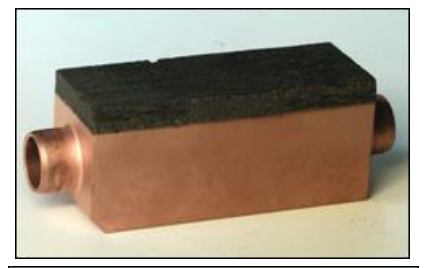

CFC flat tile mock-up

active metal casting of CFC (silicon doped NS31)

e-beam welding to $\mathrm{CuCrZr}$ heat sink

HHF fatigue testing: 1000 cycles @ 19 MWm $^{-2}$

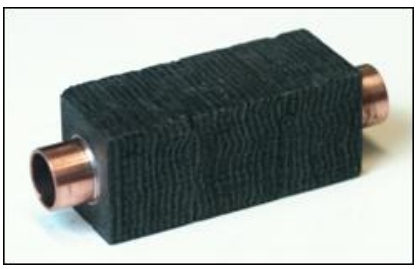

CFC monoblock drilling of CFC tiles (SEPcarb NB31) active metal casting $\left(\mathrm{AMC}^{\circledR}\right)$ low temperature HIPing

HHF fatigue testing: 1000 cycles @ 25 MWm $^{-2}$
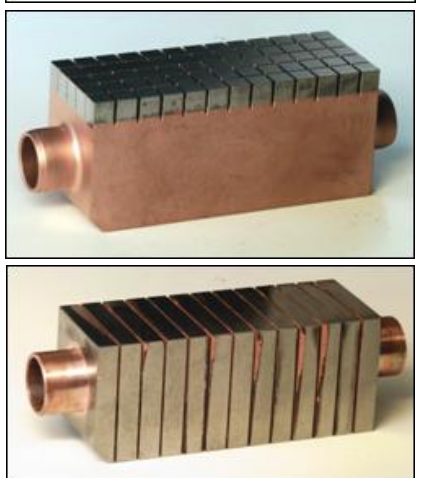

W macrobrush mock-up coating of $\mathrm{WLa}_{2} \mathrm{O}_{3}$ tiles with $\mathrm{OFHC}-\mathrm{Cu}$, e-beam welding to $\mathrm{CuCrZr}$ heat sink HHF fatigue testing: 1000 cycles @ 18 MWm $^{-2}$

\section{W monoblock mock-up} drilling of $\mathrm{W}-\mathrm{La}_{2} \mathrm{O}_{3}$ monoliths casting with $\mathrm{OFHC}-\mathrm{Cu}$ HIPing

HHF fatigue testing: 1000 cycles @ 20 MWm $^{-2}$

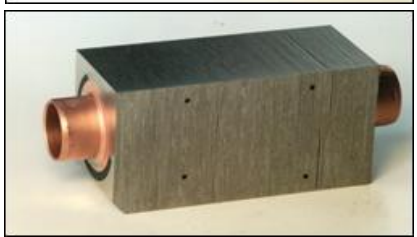

W monoblock mock-up (lamellae technique) drilling of $\mathrm{W}$ sheets casting with OFHC-Cu low temperature HIPing HHF fatigue testing: 1000 cycles @ 18 MWm $^{-2}$

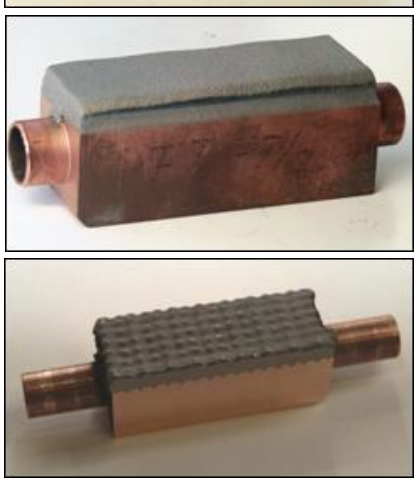

PS-W mock-up

vacuum plasma spraying of tungsten

CuCrZr heat sink

HHF fatigue testing: 1000 cycles @ 5.5 MWm ${ }^{-2}$

\section{PS-Be mock-up}

vacuum plasma spraying of beryllium (5 mm thick) manufactured by R. Castro, Los Alamos Nat. Lab. HHF fatigue testing: 1000 cycles @ 3.0 MWm ${ }^{-2}$

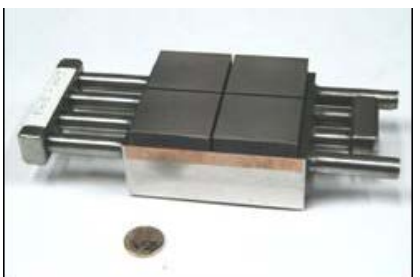

Primary first wall mock-up Be tiles $\left(42 \times 47 \times 10 \mathrm{~mm}^{3}\right)$ $\mathrm{CuCrZr}$ heat sink $(10 \mathrm{~mm})$ with $316 \mathrm{~L}$ coolant tubes $316 \mathrm{~L}$ backing plate $(30 \mathrm{~mm})$ HHF fatigue testing: 1000 cycles @ 1.5 MWm ${ }^{-2}$

Fig. 5. Survey of small scale mock-ups with different plasma facing armour (CFC, tungsten, beryllium) and different design options (flat tile components, monoblock design and plasma sprayed modules) [8] 
Similar to the divertor applications, precipitation hardened copper (CuCrZr) has also become a promising candidate for the heat sink in first wall components. Hence, additional efforts have been allocated to the development and thermo-mechanical testing of beryllium/CuCrZr-joints. Best performances obtained so far with HHF tests in the electron beam test facility JUDITH on a variety of components produced with different joining parameters have shown detachments of the Be tiles after cyclic operation only for heat fluxes $>2.75 \mathrm{MW} / \mathrm{m}^{2}$.

HHF testing has also been performed on flat $\mathrm{CuCrZr}$ heat sink modules which were coated in a plasma spray process with tungsten (see Fig. 5). These modules have shown a favourable thermal fatigue performance with peak heat loads of $5.5 \mathrm{MWm}^{-2}$ without detectable failure. Becoated component which have also been produced by plasma spray techniques didn't show any degradation of the heat removal efficiency up to $3 \mathrm{MWm}^{-2}$; however, some cracks developed perpendicular to the component's surface (i.e. parallel to the heat flux direction). These findings were predictable since both types of plasma sprayed components have not been castellated so far.

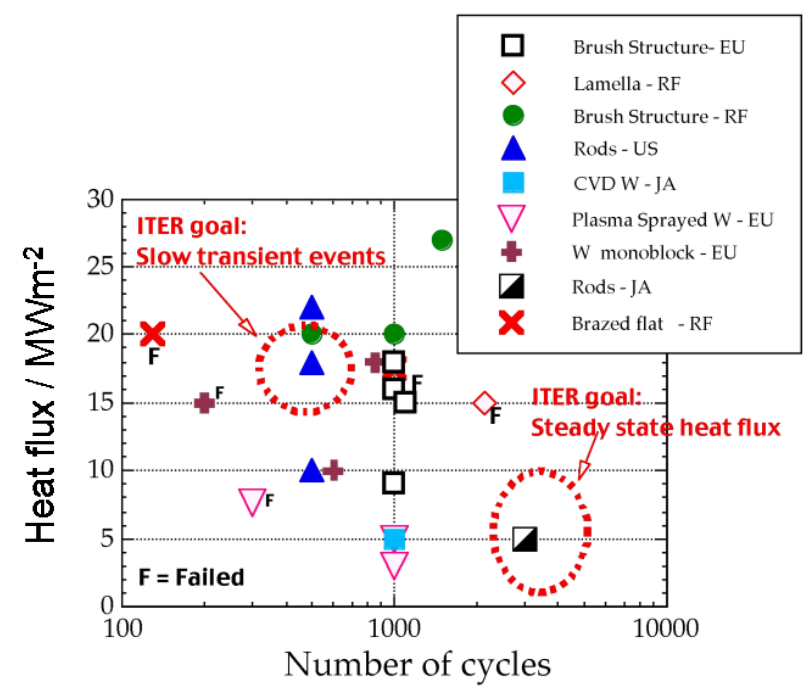

Fig. 6 Damage limits for plasma facing components with tungsten armour [cf. ITER web-page]

To compare the results form different thermal fatigue tests on plasma facing components with carbon armour which have been performed so in different laboratories in Europe, Russia and Japan, the maximum acceptable heat flux for a given pulse number is plotted in Fig. 6. This diagram also shows the ITER target values (dotted circles) for steady state operation (approx. $5 \mathrm{MWm}^{-2}$ ) and for slow thermal transients (up to $20 \mathrm{MWm}^{-2}$ ). Although HHF tests with high cycle number $(\mathrm{n}>1000)$ are scarce (mainly because they are rather time and cost consuming), the diagram clearly proves the existence of technically mature design solutions for the high heat flux components in large scale fusion devices such as ITER.

Most of the thermal load tests so far have been performed on small-scale modules [12 - 13]. These components typically have cross-sectional geometries which are identical with the proposed design solutions for PFCs in ITER or other large fusion devices; however, to minimize the manufacturing cost and to facilitate the testing procedure, in general short single-tube mock-ups with a length of about 5 to $20 \mathrm{~cm}$ have been tested. To benchmark the performance real scale modules with the actual length and assembly of the ITER divertor, medium and full scale prototypes (Fig. 7) have been manufactured and tested successfully under cyclic thermal loads in a powerful high heat flux test facility in France (FE 200) [14]. In a similar way full scale tests on a first wall panel $(\mathrm{L} \approx 1 \mathrm{~m})$ with beryllium tiles brazed to a dispersion strengthened heat sink are on the way [15].
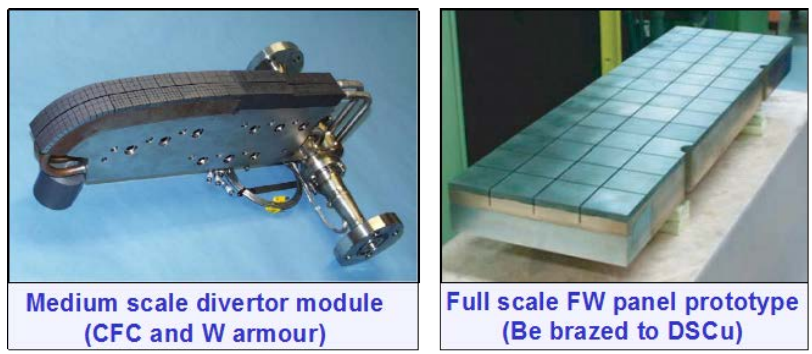

Fig. 7 Medium scale mock-up for the divertor and the first wall of ITER

\section{THERMAL SHOCK LOADING}

Beside the normal operation scenarios, transient loading conditions also have been taken into consideration $[16,18]$. Among these events (cf. table I) the so-called vertical displacement events (i.e. the malfunction of the plasma positioning system) may result in severe surface damage due to short term $(100-300 \mathrm{~ms})$ thermal loads to plasma facing components. Such an event with a deposited energy density of about $60 \mathrm{MJm}^{-2}$ (ITER) will mainly affect the surface of components with metallic PFMs (beryllium or tungsten). The material performance during these short term events is shown schematically in Fig. 8, both for metallic (e.g. tungsten or beryllium) and carbon based materials (e.g. graphites or CFC). The energy density during plasma disruptions or VDEs in general exceeds the melting threshold, not only for beryllium but also for tungsten. Depending on the energy density of the incident beam pulse, the liquefied material will either remain in the position where it is formed and recrystallize after a short period, or it will be ejected due to the high vapour pressure at the surface of the melt pool. A further increase of the incident power density may also result in a boiling and 
bubble formation of the melt layer. These processes are a major source for the formation of metallic droplets, particularly if additional (e.g. magnetic) forces are acting on the melt layer. These droplets might contaminate the plasma. Metallic dust originating from recrystallized melt droplets has been identified as a critical safety issue in future fusion devices.
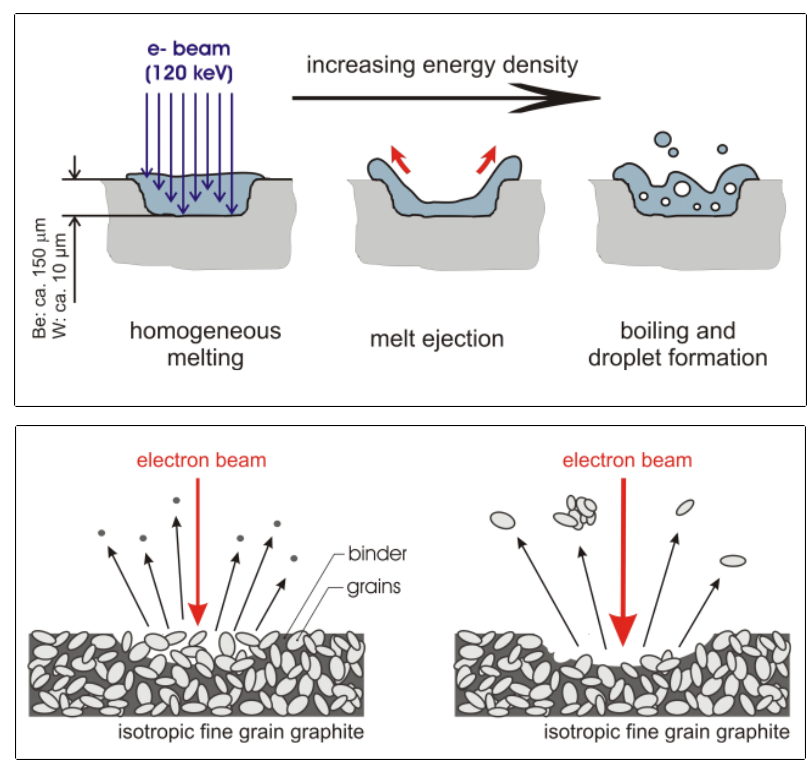

Fig. 8 Metals (top) and graphite (bottom) under intense thermal loads in an electron beam device [10].

The melt layer thickness under these events was determined experimentally in electron beam tests and was found to be in the order of a few millimetres (depending on the pulse duration), see Fig. 9. Mock-ups with un-doped CFC armour are more resistant under identical thermal loads since pure carbon does not form any liquid phase; however, some thermal erosion by sublimation and brittle destruction (see below) has been detected.

More serious material damage is expected during plasma disruptions which occur on a millisecond timescale. For ITER about $10 \%$ of the discharges are supposed to be terminated in a plasma disruption. The published data about the expected amount of deposited energy density show some scatter; furthermore, part of the incident plasma energy is absorbed by a dense cloud of ablation vapour which forms above the heat affected surface area. Nevertheless, an absorbed energy density of several $\mathrm{MJm}^{-2}$ will be deposited on the PFC surface. Due to the rather short pulse duration $(\Delta \mathrm{t} \approx 1 \mathrm{~ms}$ ) heat conduction into deeper parts of the PFM does not play any important role and the mayor damage is restricted to a thin surface layer with a thickness of several ten microns. Under these conditions metallic plasma facing materials such as beryllium or tungsten will melt instantaneously; this mechanism is associated with the formation of bubbles in the melt layer and with the ejection of metallic droplets which finally will contaminate the plasma boundary layer or will be deposited in the form of metallic dust or layers in gaps behind the PFCs. From a safety point of view this process may generate non negligible amounts of toxic beryllium particles or highly activated tungsten dust which might need periodical removal to avoid the accumulation of critical dust concentrations.

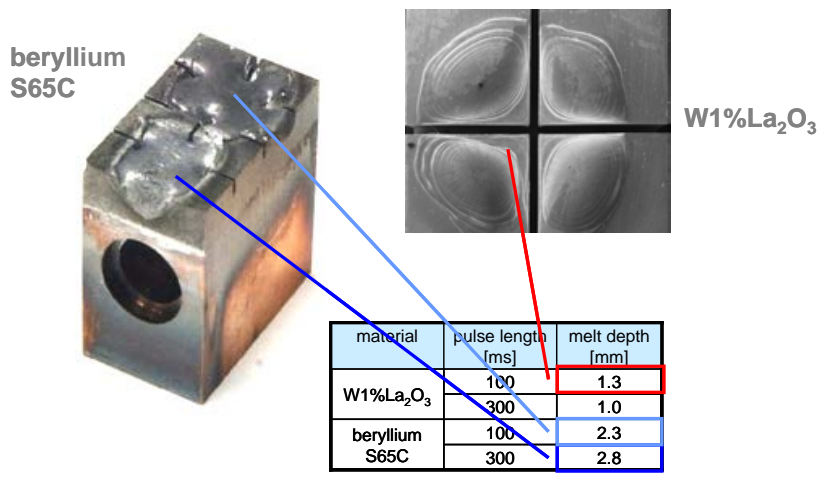

Fig. 9 Electron beam simulation of vertical displacement events with a deposited energy density of $60 \mathrm{MJm}^{-2}[10]$

The short pulse duration of disruption events will generate steep thermal gradients in the surface of the plasma facing material; this will induce severe thermal stresses which may generate cracks with a depth of several hundred microns and beyond. This effect is of special importance if the temperature of the heat effected material is below DBTT (ductile brittle transient temperature), i.e. at below $\approx 400^{\circ} \mathrm{C}$ for un-irradiated sintered tungsten.

In contrast to metallic PFMs carbon based materials such as graphites or CFCs do not melt; hence, the formation of dust particles via the above mentioned mechanism does not occur. However, brittle destruction (BD) [19], i.e. generation of thermally induced microcracks in the surface of these materials during intense thermal loads will result in the formation of carbon dust particles, if a critical threshold value of the incident beam power is exceeded (see Fig. 8, bottom). The brittle destruction mechanism has been detected in electron beam simulation experiments; a typical example is shown in Fig. 10 for an absorbed power density of $\geq 3.3 \mathrm{GWm}^{-2}(\Delta \mathrm{t}=2 \mathrm{~ms})$. Here the trajectories of the hot carbon particles are clearly visible; particle velocities $\geq 150$ $\mathrm{ms}^{-1}$ have been determined by optical time-of-flight measurements [20]. Below a critical threshold value $\left(<3 \mathrm{GWm}^{-2}\right)$ no particle emission has been observed. Up to a 2nd threshold value mainly small and medium sized particles are ejected from the surface of the plasma facing material. In fine grain graphite this process is characterized by the release of the binder phase between the graphitic grains (cf. Fig. 8, bottom). If the 2nd threshold value is 
exceeded large dust particles (grains or grain clusters) are emitted from the surface. Major concern of the carbon dust is the co-deposition together with tritium in gaps or in remote areas behind the divertor structure. In particular the large particle emission results in a substantial erosion of the graphite surface; this has been clearly demonstrated by weight loss and SEM analyses [19].
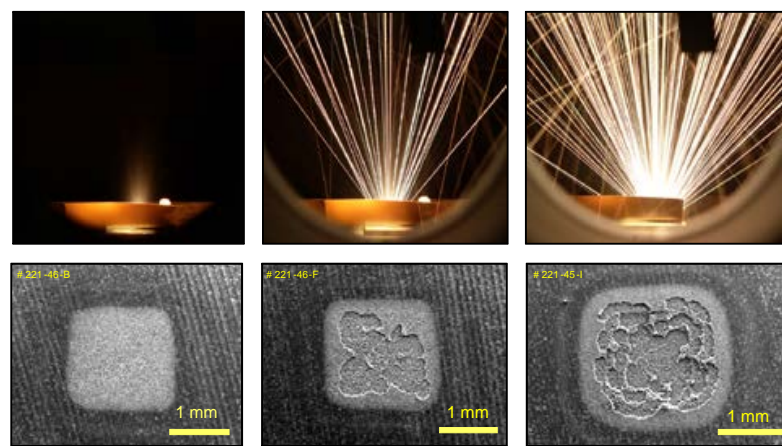

$P_{\text {abs }}=3.3 \mathrm{GW} \mathrm{m} \mathrm{m}^{2}$

$\mathrm{U}_{\mathrm{acc}}=120 \mathrm{kV}, \Delta \mathrm{t}=\mathbf{2} \mathrm{ms}$

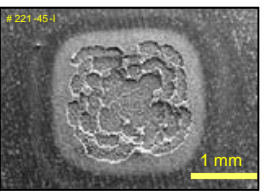

$P_{a b s}=4.3 \mathrm{GW} \mathrm{m} \mathrm{m}^{2}$

$P_{a b s}=3.1 \mathrm{GW} \mathrm{m}^{-2}$

Fig. 10 Brittle destruction of isotropic fine grain graphite (power density $\mathrm{P}_{\mathrm{abs}}=3.2 \ldots 4.3 \mathrm{GWm}^{-2}, \Delta \mathrm{t}=2 \mathrm{~ms}$,).

The threshold values for the onset of brittle destruction have been determined for graphites and CFCs both for disruption and VDE specific pulse durations, i.e. for 1 to 5 and for $100 \mathrm{~ms}$; similar studies for the ELM regime are on the way. The thermal loads during plasma disruptions and VDEs in ITER are clearly above the threshold values for brittle destruction, while the ELM regime seems to remain in a safe operation regime. Nevertheless, brittle destruction may also play an important role for ELM specific loads because of the high frequency of these events $(1 \mathrm{~Hz})$ and an integrated number of several million incidents during the lifetime of the divertor target in ITER.

Carbon dust particles have been collected and analysed by different methods. The size of these objects covers a rather wide range from a few nanometers to a maximum of about $100 \mu \mathrm{m}$, i.e. their dimensions are ranging from nanotubes to graphitic grains or even grain clusters. Simulation tests with carbon fibre composites show a rather similar behaviour compared to fine grain graphites, however, the threshold values are slightly shifted to higher energy densities; this is due to the improved thermal conductivity of this material. The material erosion strongly depends on the architecture of the CFC composite and on the type and orientation of the fibres used.

\section{NEUTRON INDUCED MATERIAL DEGRADATION}

The irradiation induced degradation of mechanical and thermal properties has been performed on selected plasma facing materials which have been subjected to ITER relevant neutron fluxes in fission type material test reactors, such as the high flux materials test reactor (HFR in Petten, The Netherlands). Furthermore, modifications in the high heat flux performance have been investigated in electron beam tests on neutron irradiated small scale components with CFC, tungsten and beryllium armour.

The heat removal efficiency of actively cooled components mainly depends on the thermal conductivity $\lambda$ of the materials. This parameter was determined in laser flash experiments which allows a direct measurement of the thermal diffusivity $\alpha$ in combination with additional recordings of the material density $\rho$ and the temperature dependent specific heat $c_{p}\left(\lambda(T)=\alpha(T) \cdot \rho(T)^{\circ} c_{p}(T)\right)$.

Carbon based materials show a rather strong decrease in thermal conductivity even after relatively low neutron fluences [21, 22]. The ITER candidate CFC armour material NB31 for example exhibits excellent thermal conductivities before neutron irradiation. Fig. 11 shows laser flash data measured in the high thermal conductivity direction (i.e. parallel to the pitch fibre reinforcement) with $\mathrm{RT}$ values exceeding $300 \mathrm{Wm}^{-1} \mathrm{~K}^{-1}$. Even low neutron fluences have a strong effect on the thermal conductivity with values below $50 \mathrm{Wm}^{-1} \mathrm{~K}^{-1}$ at room temperature. $\mathrm{n}$ irradiation to $1.0 \mathrm{dpa}$ finally results in a reduction of $\lambda$ by one order of magnitude. Due to annealing effects the thermal conductivity reduction diminishes at elevated temperatures.

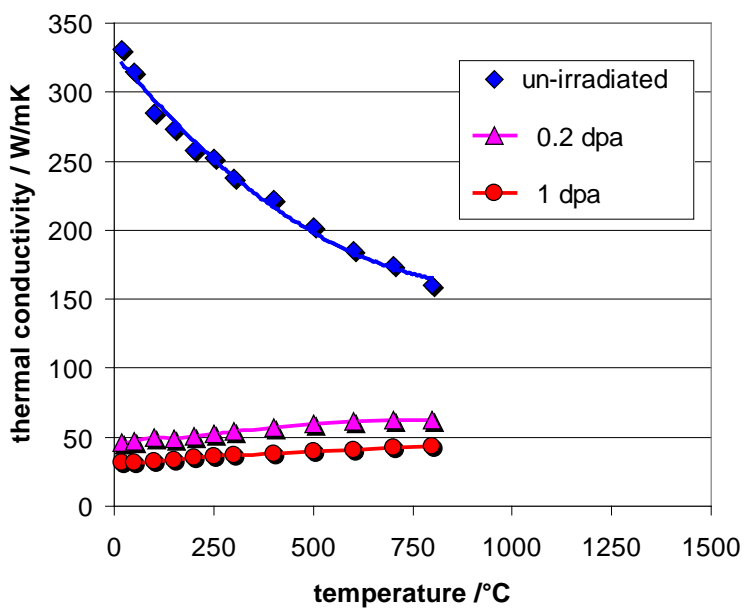

Fig. 11 Thermal conductivity of NB31 before and after neutron irradiation $\left(0.2\right.$ and $1.0 \mathrm{dpa}, \mathrm{T}_{\text {irr }}=200^{\circ} \mathrm{C}$, pitch fibre orientation) [8] 


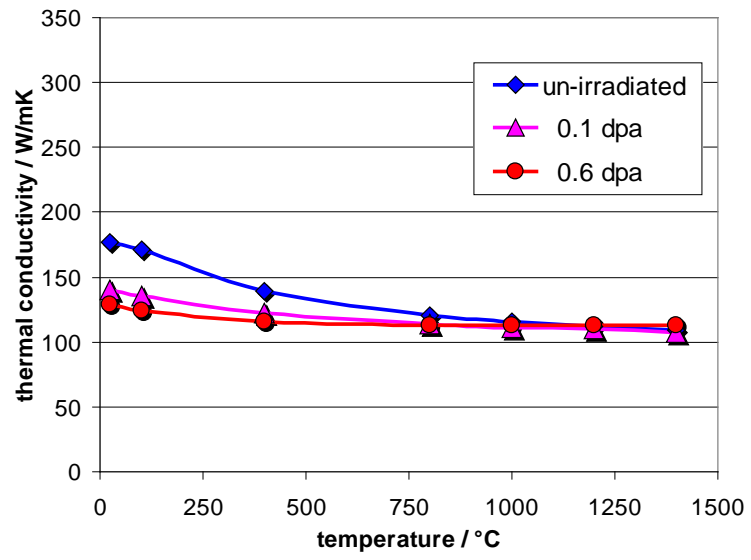

Fig. 12 Thermal conductivity of $\mathrm{W}$ before and after neutron irradiation $\left(0.1\right.$ and $\left.0.6 \mathrm{dpa}, \mathrm{T}_{\text {irr }}=200^{\circ} \mathrm{C}\right)$ [8]

The room temperature thermal conductivity of sintered tungsten is significantly smaller compared to NB31 (cf. Fig. 12); however, there is only a marginal reduction at elevated temperatures. For irradiated tungsten the neutron induced degradation of the thermal conductivity $\lambda$ is also less pronounced; in a temperature range $\mathrm{T} \leq 1400^{\circ} \mathrm{C}$ and up to the ITER specific fluence of approx. $0.6 \mathrm{dpa} \lambda$ remains well above $100 \mathrm{Wm}^{-1} \mathrm{~K}^{-1}$. For $\mathrm{T} \geq 1000^{\circ} \mathrm{C}$ the difference between irradiated and un-irradiated material is negligible.

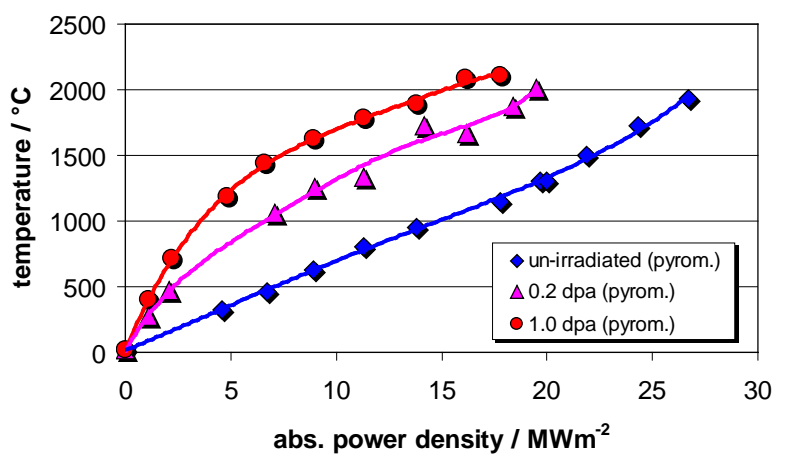

Fig. 13 Surface temperature of flat-tile divertor components with CFC armour as a function of the applied thermal load (unirradiated and neutron irradiated at $200^{\circ} \mathrm{C}$, 0.2 and $1.0 \mathrm{dpa}$ ) [8].

Actively cooled divertor components with CFC and tungsten armour (flat tile and monoblock design) have been exposed to similar neutron doses in the HFR reactor. The thermal fatigue behaviour of all mock-ups has been evaluated without and after neutron irradiation. Typical results for CFC flat tile components at different neutron fluences of 0.2 and 1.0 dpa @ $200^{\circ} \mathrm{C}$ are plotted in Fig. 13. To avoid excessive carbon vaporization these experiments were limited to surface temperatures of above $2000^{\circ} \mathrm{C}$. In compliance with these restrictions the un-irradiated components have been exposed to heat loads of more than $25 \mathrm{MWm}^{-2}$ (screening tests); after neutron irradiation these limits were achieved already below $20 \mathrm{MWm}^{-2}$. For temperatures below approx. $1000^{\circ} \mathrm{C}$ the slope of the plotted curves in Fig. 13 shows the neutron irradiation induced changes in the heat removal efficiency. For higher thermal loads, i.e. when the surface temperature exceeded values of approx. $1000^{\circ} \mathrm{C}$, part of the neutron induced defects recover.

Beside screening tests with small cycle numbers, thermal fatigue experiments have been performed with $n=1000$ cycles [8] in agreement with the experiments on un-irradiated components in chapter 4 . The results which have been obtained so far can be summarized as follows [21, 23]:

- CFC flat tiles have been exposed to cyclic thermal loads up to $15 \mathrm{MWm}^{-2}$ (at $0.2 \mathrm{dpa}$ and $1.0 \mathrm{dpa}$ ) and for 1000 thermal cycles without any failure,

- CFC monoblocks have been tested up to $12 \mathrm{MWm}^{-2}$ for 1000 cycles; screening tests performed at $14 \mathrm{MWm}^{-2}$ have been terminated caused by vaporization losses due to high surface temperatures,

- tungsten monoblock modules did not show any failure up to $18 \mathrm{Wm}^{-2}$ (0.1 and $0.6 \mathrm{dpa}$ ).

- tungsten flat tiles (macrobrush) withstood 1000 cycles at $10 \mathrm{MWm}^{-2}$ (0.1 and $\left.0.6 \mathrm{dpa}\right)$; the fatigue tests were characterized by a non-negligible increase of the surface temperature.

Neutron irradiation experiments with beryllium armoured primary first wall mock-ups (low temperature irradiation at $0.6 \mathrm{dpa}$ ) are in preparation.

\section{SUMMARY}

The design activities for the divertor and the primary first wall modules follow roughly the same general pattern which is shown schematically in Fig. 14. The major steps of the $R \& D$ activities include the design selection, the qualification of the materials for the plasma facing armour and for the heat sink, the development and improvement of reliable joining techniques. Step-by-step iterations resulted in the production of numerous small scale mock-ups which were subjected to non-destructive qualification tests and to extensive high heat flux testing, preferably in electron beam test devices. In a further step, selected material samples and small-scale modules were irradiated in material test reactors to ITER specific fluences. Finally, medium and full-scale components have been manufactured mainly by industry, but also by research laboratories. These prototype components have been exposed to cyclic thermal loads (divertor) or are now ready for fatigue performance testing (blanket modules). 


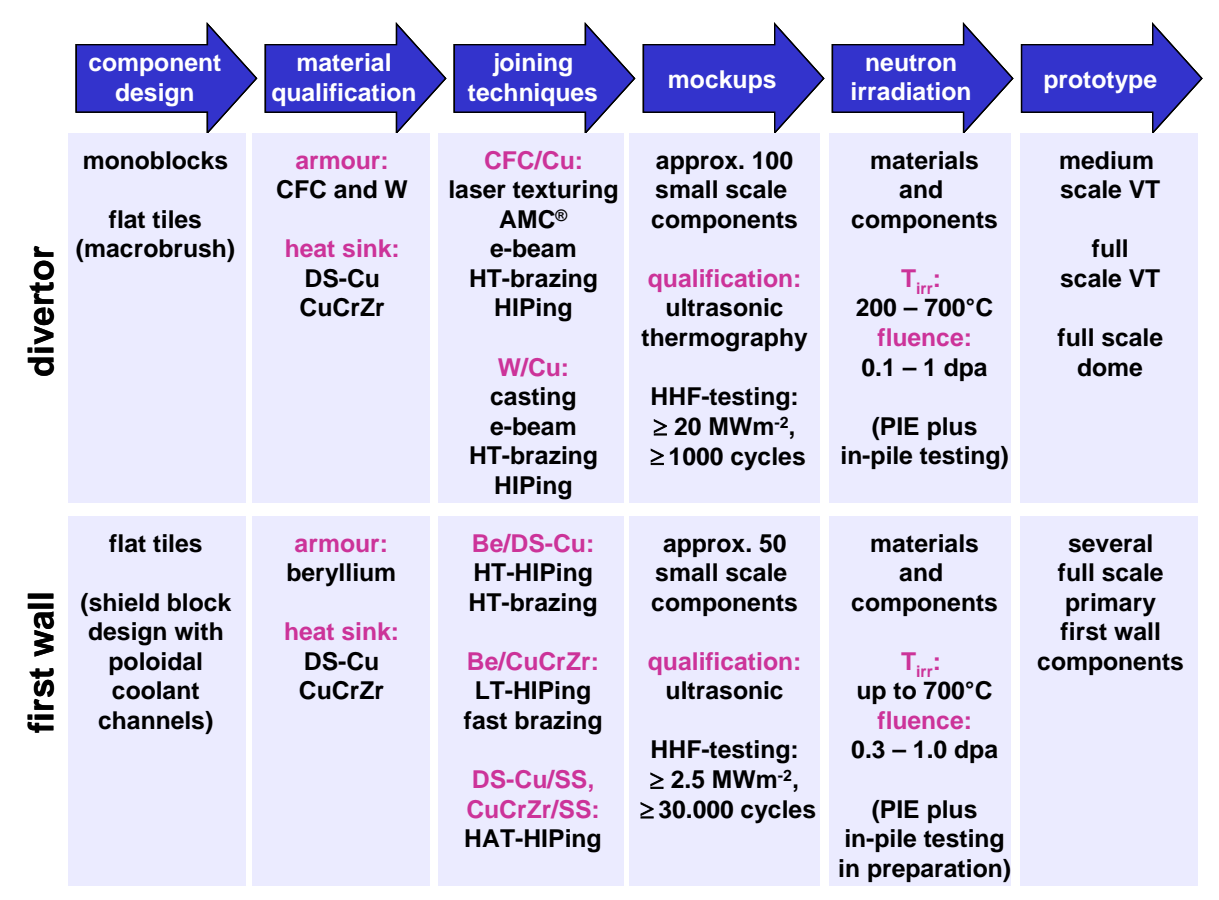

Fig. 14 Schematic presentation of the step-by-step development of first wall and divertor targets [8]

During the past few years the present design of the ITER divertor has received a well-engineered, technically mature status; this has largely been achieved by an intense collaboration within the European associations, with other international partners and with industry. In the frame of this study the relevant armour and heat sink materials have been qualified; the development and qualification of two different design options, the monoblock and the flat tile geometry have been pursued in parallel approaches. In addition, a wide spectrum of different joining methods such as e-beam welding, high temperature brazing or hot isostatic pressing (HIP) have been applied to the most promising material candidates. The quality of the bond has been benchmarked by non-destructive analyses or extensive high heat flux experiments (thermal fatigue testing and simulation of transient events). Finally medium and full scale components with tungsten and CFC armour have been evaluated successfully under ITER specific thermal loads. Today, fatigue resistant high heat flux components for thermal loads up to $20 \mathrm{MWm}^{-2}$ are technical feasible. A similar approach has been applied to develop thermal fatigue and radiation resistant first wall components. Here the low- $\mathrm{Z}$ material beryllium is the first choice for ITER; other existing or next step fusion devices also utilize or suggest carbon based materials (isotropic fine-grain graphites) and/or plasma sprayed boron carbide or tungsten coatings. Qualified heat sink materials are precipitation hardened or dispersion strengthened copper alloys; stainless steel, in particular low activation grades, may also play an important role in the longer run.

Finally, neutron irradiation experiments have been performed in material test reactors to characterise the materials degradation. Here mainly thermal and mechanical properties have been investigated under ITER specific conditions, i.e. for neutron wall loads up to $1 \mathrm{dpa}$. Under these conditions rather serious degradation effects has been identified for carbon based materials; here the thermal conductivity shows a significant decrease up to one order of magnitude, even for neutron doses as low as $0.2 \mathrm{dpa}$. A number of qualification tests have been done to evaluate the HHF performance of actively cooled high heat flux components, mainly with carbon and tungsten armour. The results of these tests clearly indicate that technically mature solutions for high heat flux components in next step thermonuclear fusion devices are feasible.

\section{REFERENCES}

[1] J. Linke: "Plasma facing materials and components for future fusion devices - development, characterization and performance under fusion specific loading conditions" to be published in Physica Scripta, 2005 
[2] R. Aymar and International Team: "ITER status, design, and material objectives", J. Nucl. Mater. 307 - 311, 1 (2002)

[3] H. Bolt et al.: "Plasma facing and high heat flux materials - needs for ITER and beyond", J. Nucl. Mater. 307 - 311, 43 (2002)

[4] M. Merola, M. Akiba, V. Barabash, I. Mazul, "Overview on fabrication and joining of plasma facing and high heat flux materials for ITER", J. Nucl. Mater, 307 - 311, 1525 (2002)

[5] H. Bolt, V. Barabash, W. Krauss, J. Linke, R. Neu, S. Suzuki, N. Yoshida, ASDEX Upgrade team, "Materials for the plasma facing components of fusion reactors", J. Nucl. Mater. 329 - 333, 66 (2004)

[6] R. Tivey et al, "ITER divertor, design issues and research and development", Fusion Eng. Design 46, 207 (1999)

[7] M. Scheerer, H. Bolt, A. Gervash, J. Linke, I. Smid: "The design of actively cooled plasmafacing components", Physica Scripta T91, 98 (2001)

[8] J. Linke, P. Lorenzetto, P. Majerus, M. Merola, D. Pitzer, M. Rödig: "EU Development of High Heat Flux Components", Proc. ANS 16th Topical Meeting on the Technology of Fusion Energy, Madison, Wisconsin, 14. - 16.09.2004

[9] I. Smid, "Material Processing and Testing of Plasma-Interactive Components for Fusion Energy Systems”, Material Science Forum Vols. 475 479, 1355 (2005)

[10] J.M. Linke, T. Hirai, M. Rödig, L.A. Singheiser, 'Performance of plasma-facing materials under intense thermal loads in tokamaks and stellarators', Fus. Sci. Tech., 46, 142 (2004)

[11] J. Schlosser et al.: 'Technologies for ITER divertor vertical target plasma facing components', Nucl. Fusion 45, 512 (2005)

[12] T. Hirai, K. Ezato, P. Majerus: 'ITER Relevant High Heat Flux Testing on Plasmas Facing Surfaces', Materials Transactions, Vol. 46, No.3, 412 (2005)

[13] H. Greuner, B. Böswirth, J. Boscary, G. Hofmann, B. Mendelevitch, H. Renner, R. Rieck: "Final design of W7-X divertor plasma facing components - tests and thermo-mechanical analysis of baffle prototypes", Fus. Eng. Design 66 - 68, 447 (2003)

[14] M. Merola, J. Palmer and the EU ITER Participating Team: 'EU Activities in Preparation of the Procurement of the ITER Divertor', Proc. Intern. Symposium on Fusion Nuclear Technology, Tokyo, 22. - 27.05.2005

[15] P. Lorenzetto, B. Boireau, C. Boudot, Ph. Bucci, A. Furmanek, K. Ioki, J. Liimatainene, A. Peacock, P. Sherlock, S. Tâhtinen, "Manufacture of Blanket Shield Modules for ITER", Proc. SOFT-23, Venice, Italy (2004), to be published

[16] G. Federici et al.: "Key ITER plasma edge and plasma-material interaction issues", J. Nucl. Mater, 313 -316, 11 (2003)

[17] J. Jacquinotet al.: "Recent developments in steadystate physics and technology of tokamaks in Cadarache", Nucl. Fusion 43, 1583 (2003)

[18] A. Zhitlukhin, et al: "Effect of ELMs and disruptions on ITER divertor armour materials" J. Nucl. Mater, 337 - 339, 684 (2005)

[19] J. Linke, S. Amouroux, E. Berthe, W. Kühnlein, M. Rödig: "Brittle destruction of carbon based materials in transient heat load tests", Proc. 22nd Symposium on Fusion Technology, Helsinki, 08. 13.09.2002

[20] T. Hirai, J. Linke, W. Kühnlein, G. Sergienko, S. Brezinsek, "Light emission from carbon-based materials under ITER relevant thermal shock loads", J. Nucl. Mater. 321, 110 (2003)

[21] M. Roedig, W. Kuehnlein, J. Linke, D. Pitzer, M. Merola, E. Rigal, B. Schedler, E. Visca: "Post irradiation testing of samples from the irradiation experiments PARIDE 3 and PARIDE 4", J. Nucl. Mater. 329 - 333, 766 (2004)

[22] V. Barabash, G. Federici, J. Linke, C.H. Wu: "Material/plasma surface interaction issues following neutron damage", J. Nucl. Mater. 313 316, $42-51$ (2003)

[23] M. Rödig et al.: "High Heat Flux Performance of Neutron Irradiated Plasma Facing Components", J. Nucl. Mater. 307 - 311, 53 (2002) 